\title{
A rare complication of uterine leiomyomata: Severe haemoperitoneum caused by the rupture of an overlying subserosa variceal vein: A case report
}

\author{
RAZVAN POPOVICI $^{1,2}$, ANDA PRISTAVU ${ }^{1,2}$, DAN-CONSTANTIN ANDRONIC ${ }^{3}$, PAULA ZAN ${ }^{2}$, BENEDICTE RIEY $^{2}$, \\ MURARASU MARA ${ }^{2}$, RALUCA MOGOS ${ }^{1,2}$, LOREDANA MARIA HIMINIUC ${ }^{1,2}$ and MIHAELA GRIGORE ${ }^{1,2}$ \\ ${ }^{1}$ Department of Obstetrics and Gynaecology, 'Grigore T. Popa', University of Medicine and Pharmacy, 700015 Iasi; \\ ${ }^{2}$ Department of Obstetrics and Gynaecology, 'Cuza Voda' Clinical Hospital of Obstetrics and Gynaecology, 700038 Iasi; \\ ${ }^{3}$ Department of Surgery, 'Grigore T. Popa' University of Medicine and Pharmacy, 700015 Iasi, Romania
}

Received June 8, 2021; Accepted July 8, 2021

DOI: $10.3892 / \mathrm{etm} .2021 .10804$

\begin{abstract}
The rupture of a subserosa variceal vein overlying a uterine myoma is a rare complication and a less common cause of gynecologic haemoperitoneum. The literature data are scarce regarding this condition and less than 100 cases have been reported (including those occurring during pregnancy). The present case is of a 48-year-old woman, with a history of asymptomatic uterine myoma, who was hospitalised for severe abdominal pain with sudden onset and signs of hypovolemic shock. The emergency conventional imaging exams confirmed the diagnosis of uterine myoma and haemoperitoneum, but did not reveal the source of bleeding. The cause of haemoperitoneum was detected by means of emergency laparotomy. In order to obtain quick hemostasis, in the settings of a rapid deteriorating hypovolemic shock, a supracervical hysterectomy was performed. The aim of the article is to raise awareness to gynecologists regarding this extremely rare life-threatening complication of the most common benign tumor of the uterus.
\end{abstract}

\section{Introduction}

Uterine leiomyoma is the most common benign condition of the uterus. The usual complications, such as menorrhagia, dysmenorrhea, pelvic pain, and infertility, are well known and fully covered by the literature. Although the tumors may reach large dimensions, in extremely rare cases massive haemoperitoneum can occur due to the rupture of an overlying variceal

Correspondence to: Dr Loredana Maria Himiniuc, Department of Obstetrics and Gynaecology, 'Cuza Voda' Clinical Hospital of Obstetrics and Gynaecology, 34 Cuza Voda Street, 700038 Iasi, Romania

E-mail: loredanahiminiuc@gmail.com

Key words: uterine myoma, subserosa vein, rupture, haemoperitoneum, gynaecological emergency vein. Rokitanski first described the complication, following an autopsy report, in $1861(1,2)$.

The real incidence is difficult to assess and the reported cases are scarce. Performing an Embase, Scopus, and PubMed search, the number of reported cases was identified at approximately less than 100, including those occurring during pregnancy. Saidi et al reported 50 cases before 1961 (3), Akahira et al reported 7 cases between 1961 and 1997 (4), Jain et al reported no more than 7 cases from 1994 to 2004 (5), and 9 cases were revealed by the search of the present authors between 2005 and 2020 (2,6-13).

The present case study included a 48-year-old woman with massive haemoperitoneum caused by the rupture of a subserosa vein overlying a uterine leiomyoma. To the best of our knowledge, it is the first reported case in Romania, and through this, the article aim is to raise awareness of this extremely rare, but potentially fatal complication.

\section{Case presentation}

A 48-year-old G1P1 (one gestation, one parity) woman was referred to the Gynaecology Department of the 'Cuza-Voda' University Hospital, Iasi, Romania presenting abdominal pain with sudden onset, moderate genital bleeding, cold and sweat extremities, hypotension, and tachycardia. The patient declared that she has been diagnosed with uterine myoma 2 years before, during a routine gynecologic examination. She had been asymptomatic until hospital presentation. In addition, she was diagnosed 1 year earlier with mild risk hypertension, being treated with $\beta$-blockers since then. Clinical examination revealed a tender, distended abdomen with dullness in flanks, skin pallor, cold and sweat extremities, and tachycardia (124 beats/min). Blood pressure at department admittance was 90/60 mmHg, $1 \mathrm{~h}$ later it had dropped to 70/40 $\mathrm{mmHg}$. Initial haemoglobin level was $9.2 \mathrm{~g} / \mathrm{dl}$, dropping $1 \mathrm{~h}$ later to $7.1 \mathrm{~g} / \mathrm{dl}$. Transabdominal ultrasonography revealed a high amount of free fluid within the peritoneal cavity, suggestive of massive haemoperitoneum, and an enlarged uterus with an echogenic mass of $97.3 / 85.2 \mathrm{~mm}$ cranially to the uterine fundus, with posterior extension (Fig. 1). 
Emergency enhanced computed tomography scan confirmed the presence of a massive haemoperitoneum, the uterine fundal tumor resembling a myoma $(95 / 92 / 85 \mathrm{~mm})$ and presenting multiple venous dilatations with a maximum calibre of $6 \mathrm{~mm}$, overlying the tumor. No other pathologic findings were evident. Due to the continuous reduction in blood pressure in spite of the intense supportive treatment, an emergency laparotomy was decided. A sub-umbilical midline vertical incision was performed, and approximately 2,000 $\mathrm{ml}$ of blood and clots (some of them having impressive dimensions) were found. The uterus was enlarged, having a fundal myoma of $10 \mathrm{~cm}$ in the maximum diameter. Multiple venous dilatations covered the posterior part of the tumor (Fig. 2). Due to the increased angulation of the uterine arteries and subsequent reduction of uterine blood flow produced by the exteriorization of the uterus from the abdominal cavity, the bleeding source from a venous varicosity was intermittent and minimal (Fig. 3). The incision was extended laterally to the umbilicus, by a general surgeon, to allow careful search in the superior compartment of the abdomen for another source of bleeding. Since no other bleeding source was found and the variceal tear continued to bleed when the uterus was repositioned inside the abdominal cavity, the variceal rupture was determined as the source of the massive haemoperitoneum. Due to the altered hemodynamic status of the patient and haemoglobin level of $5.2 \mathrm{~g} / \mathrm{dl}$ during surgery, a supracervical hysterectomy was performed in order to achieve quick haemostasis. In the postoperative period, after the transfusion of 4 units of whole blood, the status of the patient consistently improved and the recovery was uneventful.

This case report was conducted in accordance with the World Medical Association Declaration of Helsinki. Patient consent was obtained.

\section{Discussion}

Venous congestion plays an important role in varicose venous formation. Increased abdominal pressure may lead to the rupture of superficial dilated veins, which overlie a large myoma of the uterus (5). Uterine contractions during menstrual period can create a tension effect on the blood vessels resulting in its rupture (14). Other predisposing factors for venous congestion are pregnancy, large myoma $(>10 \mathrm{~cm})(6)$, nulliparity, age between 30 and 49 years, hormonal replacement therapy, and warfarin treatment (15). Precipitating factors such as constipation, intense physical activity, coughing, and sexual intercourse (6) increase the abdominal pressure in an acute manner and may have a catalytic effect on the predisposing factors. Although the majority of studies have assessed the theories regarding the predisposing factors, Dahan and Ahmadi state that the risk of formation and rupture of the varicose veins overlying a myoma is independent of age, parity and size of the tumor (16).

In the majority of cases, patients present signs of hypovolemic shock and abdominal pain with sudden onset. The precipitating factors for the increase in abdominal pressure may be present. In women of reproductive age, in these settings, the rupture of an ectopic pregnancy or luteal cyst represents the most common diagnosis to consider. Torsion of a subserous myoma, red degeneration, avulsion and sarcomatous degeneration are common causes of acute abdomen in patients with history of uterine leiomyoma (11). Another cause of non-gynecologic haemoperitoneum must be excluded. In most cases, the actual cause of bleeding is often difficult to specify prior to the surgical procedure $(11,13)$.

Imaging techniques including ultrasonography, magnetic resonance imaging (MRI) or computer tomography can establish a positive diagnosis of haemoperitoneum and uterine myoma, in rare cases it can reveal the site of bleeding (13). Contrast-enhanced 3-dimensional MRI allows an accurate diagnosis regarding the site of the bleeding, but often it is not available in emergency settings $(13,17)$.

An acute haemoperitoneum represents an absolute emergency. In a hemodynamic unstable patient the treatment is intensive, both supportive and surgical. Blood transfusions and intravenous fluids should be administered (13). Intravascular disseminated coagulopathy is common in severe cases and for an improved outcome, it is advisable to consider the administration of recombinant factor VIIa. In our case, performing a supracervical hysterectomy decreased the operative time and blood loss, the administration of recombinant factor VIIa not being necessary. The main disadvantage of an emergency supracervical hysterectomy is represented by the impossibility of having a Pap test result prior to the surgical intervention and the patient must be included in a careful cervical screening following the procedure. Therapeutic laparoscopic approach has not described in any case report, thus far. Some authors recommend an exploratory laparoscopy in cases of acute abdomen with uncertain etiology (6). In young patients, the preservation of fertility is the main objective, but it may not be achieved in all cases (4). Hemostatic suture or myomectomy should be attempted as a first choice in women of reproductive age. However, in cases of fertility-sparing procedures, large myomas can cause serious problems regarding hemostasis. Myomectomy presents a considerable risk of persistent or recurrent bleeding from the site of myometrial repair, and in patients with important bleeding prior to surgery, this procedure may worsen their hemodynamic status (17). In cases of menopausal women, hysterectomy is advisable due to a better hemostasis and a shorter operative time. In all cases with conscious patients, they should be informed that the intraoperative findings and the hemodynamic status during surgery are essential in choosing the type of surgical procedure.

The current case is of a premenopausal patient of low parity (1P), with a positive history of uterine myoma, without any predisposing and precipitating factors. Jenayah et al mentioned that myomas $>10 \mathrm{~cm}$ have an increased risk for subserosal variceal development (6). In our case the maximum size of the tumor was $9.5 \mathrm{~cm}$, close below the limit. Of note was the fast degradation of the hemodynamic status within $1 \mathrm{~h}$ from admittance. Intraoperatively, the angulation of uterine arteries caused by uterine exteriorization from the abdominal cavity significantly reduces the blood flow of the uterus and the bleeding point from the variceal tear may be difficult to observe. Repositioning the uterus can facilitate the proper assessment of the varicosities and possible bleeding points. In case of fundal and posterior location of a large fibroid, which tends to deeply impact the uterus within the sacral concavity, as in the case in the present study, disimpaction manoeuvres 


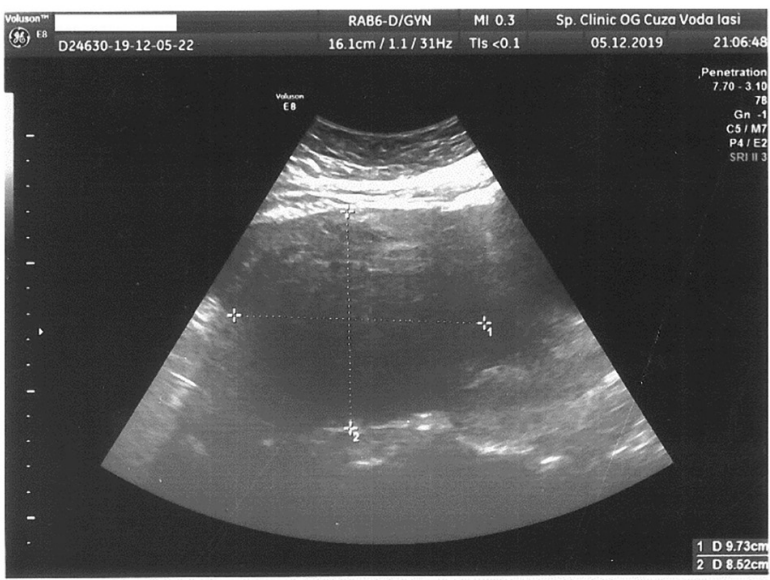

Figure 1. Ultrasonographic aspect of the myoma located at the fundus of the uterus.

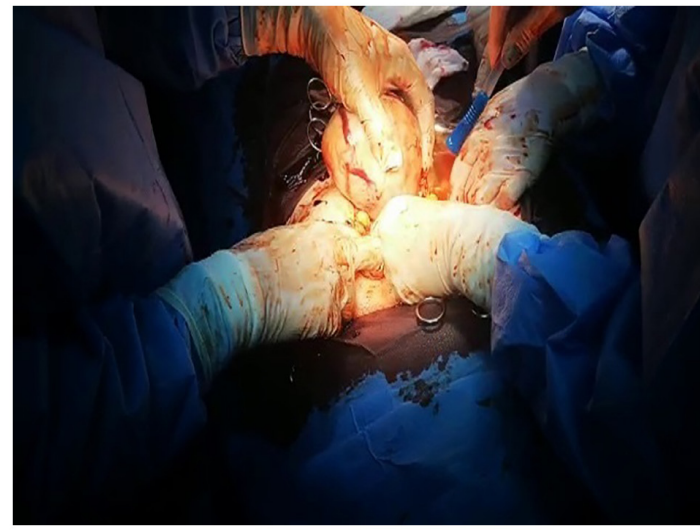

Figure 2. Multiple venous varicosities apparently without any bleeding spots after the externalization of the uterus.

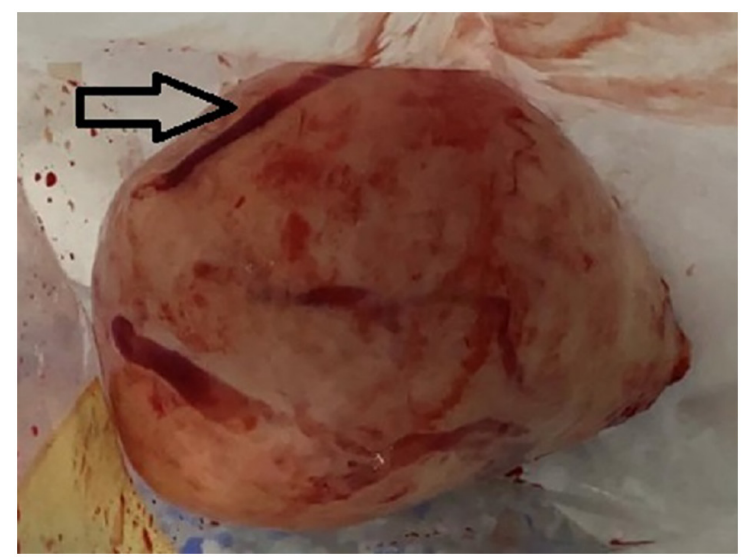

Figure 3. Bleeding point. Arrow indicates that the posterior part of the uterus is facing upward.

of a less mobile uterus can modify the uterine artery flow and momentarily stop the bleeding. A careful inspection followed by saline irrigation of the abdominal cavity, is recommendable for the exclusion of other possible bleeding sites. The presented case of a massive haemoperitoneum caused by the rupture of a subserosa vein overlying a uterine leiomyoma is, to the best of our knowledge, the first reported in Romania, and through this article the aim is to raise awareness of this extremely rare, but potentially fatal complication.

\section{Acknowledgements}

Not applicable.

\section{Funding}

No funding was received.

\section{Availability of data and materials}

The data used and/or analyzed during the current study are available from the corresponding author on reasonable request. Supplementary data are available from the corresponding author.

\section{Authors' contributions}

RP, MG, and AP designed the study and wrote the manuscript. PZ, MM, BR, DCA, RM, and LMH performed the literature research and interpreted the data. All authors have read and agreed to the published version of the manuscript. RP, AP and MG confirm the authenticity of all the raw data.

\section{Ethics approval and consent to participate}

This case report was conducted in accordance with the World Medical Association Declaration of Helsinki. Patient consent was obtained.

\section{Patient consent for publication}

Not applicable.

\section{Authors' information}

Loredana Maria Himiniuc: https://orcid.org/0000-00024721-4642.

\section{Competing interests}

The authors declare that there are no competing interests.

\section{References}

1. Buttery BW: Spontaneous haemoperitoneum complicating uterine fibromyoma. Aust N Z J Obstet Gynaecol 12: 210-213, 1972.

2. Porpora MG, Musacchio L, Piacenti I, D' Alessandris N, Pecorini F, Iafrate F and Benedetti Panici P: Massive haemoperitoneum caused by uterine leiomyoma: A case report. J Obstet Gynaecol 40: 735-736, 2020.

3. Saidi F, Constable JD and Ulfelder H: Massive intraperitoneal hemorrhage due to uterine fibroids. Am J Obstet Gynecol 82: 367-374, 1961.

4. Akahira J, Ito $\mathrm{K}$, Nakamura $\mathrm{R}$ and Yajima A: Massive intraperitoneal hemorrhage and hypovolemic shock due to rupture of a coronary vessel of a uterine leiomyoma: A report of two cases. Tohoku J Exp Med 185: 217-222, 1998.

5. Jain P, Pradhan P, Cietak KA and Anyanwu L: Acute abdomen following spontaneous variceal rupture overlying uterine leiomyoma. J Obstet Gynaecol 24: 589, 2004. 
6. Jenayah AA, Saoudi S, Sferi N, Skander R, Marzouk SB, Cherni A, Sfar E, Chelli D and Boudaya F: Spontaneous subserosal venous rupture overlying a uterine leiomyoma in a young woman. Pan Afr Med J 28: 205, 2017.

7. Ihama Y, Miyazaki T and Fuke C: Hemoperitoneum due to rupture of a subserosal vein overlying a uterine leiomyoma. Am J Forensic Med Pathol 29: 177-180, 2008.

8. Althobaiti FA, Alsaadi KK and Althobaiti AA: A case of hemoperitoneum due to spontaneous bleeding from a uterine leiomyoma. Am J Case Rep 20: 167-170, 2019.

9. Rokhgireh S, Kashi AM, Kermansaravi M, Tajbakhsh B Allahqoli L, Alkatout I and Khodaverdi S: Hemoperitoneum due to bleeding from a vein overlying a subserous uterine myoma: A case report. J Med Case Rep 14: 55, 2020.

10. Elkbuli A,Shaikh S,McKenney Mand BonevaD: Life-threatening hemoperitoneum secondary to rupture of a uterine leiomyoma: A case report and review of the literature. Int J Surg Case Rep 61: 51-55, 2019.

11. Dasari P and Maurya DK: Hemoperitoneum associated with fibroid uterus. J Obstet Gynecol India 55: 553-554, 2005.
12. Sneha S, Munikrishna M and Sheela SR: A rare case of hemoperitoneum due to rupture of vessels over the surface of leiomyoma. J Clin Biomed Sci 9: 23-24, 2019.

13. Aydin C, Şen Selim H, Eriş S and Yalçin Y: Haemoperitoneum: An extremely rare complication of leiomyoma. J Obstet Gynaecol 35: 109-110, 2015.

14. Horowitz E, Dekel A, Feldberg D and Rabinerson D: Massive hemoperitoneum due to rupture of an artery overlying a uterine leiomyoma: A case report. Acta Obstet Gynecol Scand 84: 408-409, 2005.

15. Tang A, Rao S and Cawdell G: Massive intra-abdominal haemorrhage due to spontaneous bleeding from fibroids in a post-menopausal woman. J Obstet Gynaecol 28: 244-245, 2008.

16. Dahan MH and Ahmadi R: Spontaneous subserosal venous rupture overlying a uterine leiomyoma. A case report. J Rep Med 47: 419-420, 2002.

17. Kassegne I, Kolani K, Tchangai B, Kanassoua K, Adabra K, Alassani F, Amavi AK and Dosseh EDJ: Myomectomies for massive hemoperitoneum from spontaneous bleeding of a uterine myoma. J Surg Case Rep 2017: rjx127, 2017. 